\title{
Localization and characterization of two-dimensional targets buried in a cluttered environment
}

\author{
Anthony Dubois, Kamal Belkebir ${ }^{1}$ and Marc Saillard \\ Institut Fresnel, UMR-CNRS 6133, Campus de Saint Jérôme, case 162, \\ 13397 Marseille Cedex 20, France \\ E-mail: kamal.belkebir@fresnel.fr
}

Received 14 April 2004, in final form 20 September 2004

Published 8 November 2004

Online at stacks.iop.org/IP/20/S63

doi:10.1088/0266-5611/20/6/S05

\begin{abstract}
In the present paper, we consider a two-dimensional inverse scattering problem involving two semi-infinite media separated by an interface. The targets under test are assumed to be buried in one of the two media while the sources and the receivers are located in the other medium (limited-aspect data configuration). We present an iterative scheme to reconstruct the permittivity distribution of the unknown object. This method consists in building up a sequence of the parameter of interest by minimizing, at each iteration step, a cost functional representing the discrepancy between the data and those that would be obtained with the best available estimation of the parameter. In addition, when clutter is present, the decomposition of the time reversal operator method is used to improve the signal-to-clutter ratio, since it allows us to synthesize a wave that focuses on the scatterer. The data associated with this incident field are included in the iterative minimization procedure.
\end{abstract}

(Some figures in this article are in colour only in the electronic version.)

\section{Introduction}

The use of probing waves for the characterization of buried objects covers a wide range of potential applications, in medical imaging, civil engineering, subsoil probing, etc. In many cases, non-invasive techniques are required and an interface prevents the transmitters and the receivers to surround the area of interest. In this paper, we focus on such an 'aspectlimited' configuration, where a flat interface separates the homogeneous medium containing the antennas, namely air, from another semi-infinite medium surrounding the object under test. To determine the position, shape and constitutive parameters of this object, an inverse scattering algorithm based on iterative minimization of a cost function is used. This iterative method is described in [1] for the homogeneous background medium configuration. In the present

\footnotetext{
${ }^{1}$ Author to whom correspondence should be addressed.
} 
paper, the stratified configuration is taken into account via the related Green function. The expression as well as the numerical computation of this Green function can be found in [2]. This kind of inversion algorithm is not very robust against clutter resulting from small-scale random fluctuations of the permittivity, like those one may encounter when probing media like subsoil or concrete. In such conditions, spurious bright scatterers appear in the reconstruction, especially at the boundary of the imaged domain, to mimic the contributions of inhomogeneities located away from this domain.

To improve the signal-to-clutter ratio and, consequently, the robustness and the accuracy of the algorithm, it is suggested in this paper to use the DORT (decomposition of the time reversal operator) method [3], which permits one to synthesize a wave focusing onto the scatterer. In our previous work, the DORT method has been used to detect and localize scatterers embedded in the probed area, as a preprocessing before inversion. The robustness of the technique has been tested against both experimental data [4] and synthetic cluttered data [5]. The key point here is now to benefit from the ability to focus energy onto the scatterers by including the response to such an incident wave in the inversion process.

The ill-posed character of such inverse problems is now well known. For instance, it is shown that, in the time-harmonic regime, a homogeneous object embedded in a semi-infinite homogeneous background provides almost the same scattered field as a modulated medium. Therefore, depending on the initial guess, the inverse scattering algorithm may lead to strongly different solutions. To overcome this problem, one may use some a priori information or specific regularization.

In this paper, it is shown that combining the DORT method with a priori knowledge of the sign of the contrast of permittivity drastically improves the accuracy of the characterization of an object buried in a highly cluttered environment.

\section{Notation and statement of the problem}

The geometry of the problem is shown in figure 1, where a cylindrical object of arbitrary cross-section is confined in a bounded domain $\Omega$. The embedding medium is assumed to be semi-infinite and homogeneous, with permittivity $\varepsilon_{2}=\varepsilon_{0} \varepsilon_{2 r}$ and permeability $\mu=\mu_{0}$ ( $\varepsilon_{0}$ and $\mu_{0}$ being the permittivity and permeability of vacuum, respectively), separated from the upper medium $\left(\varepsilon_{1}, \mu_{0}\right)$ by a flat interface at $y=0$. The scatterers are assumed to be inhomogeneous dielectric cylinders with complex permittivity distribution $\varepsilon(\boldsymbol{r})=$ $\varepsilon_{0} \varepsilon_{r}(\boldsymbol{r})$; the entire configuration is non-magnetic $\left(\mu=\mu_{0}\right)$. The wavenumber in vacuum is denoted by $k_{0}$.

A right-handed Cartesian coordinate frame $\left(O, \boldsymbol{u}_{x}, \boldsymbol{u}_{y}, \boldsymbol{u}_{z}\right)$ is defined. The $z$-axis is parallel to the invariance axis of the scatterer. The position vector $\mathbf{O M}$ can then be written as:

$$
\mathbf{O M}=x \boldsymbol{u}_{x}+y \boldsymbol{u}_{y}+z \boldsymbol{u}_{z}=\boldsymbol{r}+z \boldsymbol{u}_{z}
$$

The sources that generate the electromagnetic excitation are assumed to be lines parallel to the $z$-axis, located at $\left(\boldsymbol{r}_{l}\right)_{1 \leqslant l \leqslant N_{s}}$ in the upper medium.

For the inverse scattering problem, we assume that the unknown object is successively illuminated by $N_{s}$ electromagnetic excitations and, for each incident field, the scattered field is available along a contour $\Gamma$ at $N_{r}$ positions. The direct scattering problem may be reformulated as two coupled contrast-source integral relations: the state (or observation) equation given by

$$
E_{l}^{\mathrm{d}}(\boldsymbol{r} \in \Gamma)=k_{0}^{2} \int_{\Omega} \chi\left(\boldsymbol{r}^{\prime}\right) E_{l}\left(\boldsymbol{r}^{\prime}\right) G_{\Gamma}\left(\boldsymbol{r}, \boldsymbol{r}^{\prime}\right) \mathrm{d} \boldsymbol{r}^{\prime}
$$




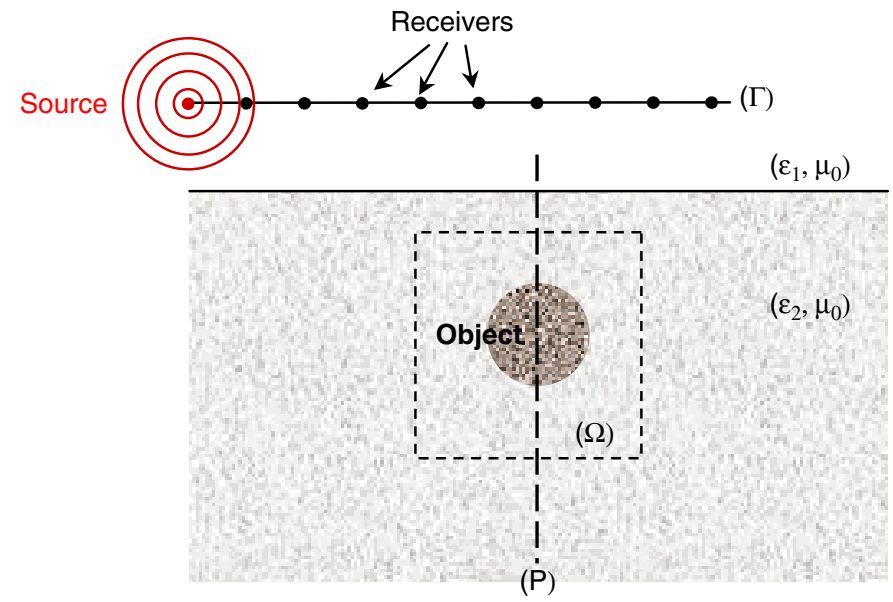

Figure 1. Geometry of the problem. An interface separating two infinite half-spaces is considered. The emitting and receiving antennas, regularly distributed along a segment $\Gamma$, are located in the upper medium, while the target under test is buried in the lower medium.

and the field (or coupling) equation given by

$$
E_{l}(\boldsymbol{r} \in \Omega)=E_{l}^{\mathrm{inc}}+k_{0}^{2} \int_{\Omega} \chi\left(\boldsymbol{r}^{\prime}\right) E_{l}\left(\boldsymbol{r}^{\prime}\right) G_{\Omega}\left(\boldsymbol{r}, \boldsymbol{r}^{\prime}\right) \mathrm{d} \boldsymbol{r}^{\prime},
$$

where $E^{\mathrm{d}}$ and $E$ represent the scattered field and the total field, respectively. $\chi(\boldsymbol{r})=\varepsilon_{r}(\boldsymbol{r})-\varepsilon_{2 r}$ denotes the permittivity contrast which vanishes outside the target and $G_{\Gamma, \Omega}\left(\boldsymbol{r}, \boldsymbol{r}^{\prime}\right)$ is the twodimensional (2D) Green's function for the configuration under consideration. Details of the expression as well as the numerical computation of this Green function can be found in [2]. For the sake of simplicity, equations (2) and (3) are rewritten in operator notation as

$$
\begin{aligned}
& E_{l}^{\mathrm{d}}=\mathbf{G}_{\Gamma} \chi E_{l}, \\
& E_{l}=E_{l}^{\mathrm{inc}}+\mathbf{G}_{\Omega} \chi E_{l} .
\end{aligned}
$$

\section{Inversion procedure}

The inverse scattering problem consists in finding the function $\chi(r \in \Omega)$ in the investigated domain $\Omega$ (test domain) so that the scattered field associated with $\chi$ matches the measured scattered field $f_{l}(\boldsymbol{r} \in \Gamma)$. Many iterative methods have been developed for solving such inverse problems. In these methods, starting from an initial guess, the parameter of interest is gradually adjusted by minimizing a cost functional involving the measured scattered field data. A brief review of the literature mainly shows two approaches, linearized methods and non-linearized ones. In the first approach, namely the Newton-Kantorovich method $[6,7]$ or the distorted-wave Born method $[8,9]$ — an analytical equivalence between these two methods is established in [10] - the field in the scattering domain is considered as fixed at each iteration step. This field is the solution of the direct problem for the best available estimation of the parameter. In the second approach, namely the modified gradient method [11] or the contrast source inversion method [12], the field is considered as an unknown that is obtained together with the parameter during the minimization procedure. When the linearized methods 
converge, they lead to a satisfactory result. However, they are sensitive to noise in the data [13]. Hence, they are usually accompanied by a regularization procedure, in particular when the objects under test are large and/or with high contrast or when noise is present. The nonlinearized methods are stable with respect to noise and therefore do not require a regularization. However, convergence is slow and the quality of the reconstruction is less satisfactory than the one obtained with the linearized method. It should be noted, however, that including a regularizer in the non-linearized methods improves the quality of the reconstructions, see [14]. A hybrid method combining ideas from the aforementioned approaches was investigated in [1], where a comparison between four non-regularized methods (linearized, non-linearized and two hybrid methods) is reported. The reconstructions were carried out using experimental data corresponding to several targets of different shapes and constitutive materials. In all cases, the

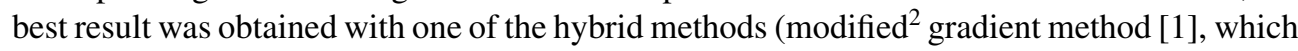
could be seen either as a variant of the modified gradient method or as a variant of a linearized method). Therefore, in the present paper, the authors use this hybrid method to characterize targets buried in a cluttered environment.

\subsection{Principles of the algorithm}

In this hybrid method, two sequences related to contrast and total field inside the test domain, $\left\{\chi_{n}\right\}$ and $\left\{E_{l, n}\right\}$, respectively, are built up according to the following recursive relations:

$$
\begin{aligned}
& E_{l, n}=E_{l, n-1}+\alpha_{l, n ; v} v_{l, n}+\alpha_{l, n ; w} w_{l, n}, \\
& \chi_{n}=\chi_{n-1}+\beta_{n} d_{n}
\end{aligned}
$$

where $v_{l, n}, w_{l, n}$ and $d_{n}$ are updating directions with respect to the total field $E_{l, n}$ and contrast, respectively, and where $\alpha_{l, n}, \beta_{l, n}$ are scalar coefficients. The updating directions $v_{l, n}$ and $d_{n}$ are chosen as the standard Polak-Ribière conjugate-gradient directions [15], while $W_{l, n}$ is given by

$$
w_{l, n}=\tilde{E}_{l, n-1}-E_{l, n-1}, \quad \tilde{E}_{l, n-1}=\left[1-\mathbf{G} \chi_{n-1}\right]^{-1} E_{l}^{\text {inc }},
$$

where $\tilde{E}_{l, n-1}$ represents the total field inside the test domain $\Omega$, calculated from the coupling equation with contrast $\chi_{n-1}$. Note that when $\alpha_{l, n ; w}=0$, the recursive relation (6) is the same as the one used in the modified gradient method, in which the coupling equation (3) is not solved at each iteration step. Conversely, when $\alpha_{l, n ; v}=0$ and $\alpha_{l, n}=1$, the recursive relation (6) reduces to $E_{l, n}=\tilde{E}_{l, n-1}$, thus leading to a linearized method such as the Newton type method. Consideration of both directions $w_{l, n}$ and $v_{l, n}$ leads to a better solution than with only one field direction. Indeed, the scalar weights $\alpha_{l, n}$ and $\beta_{n}$ are chosen at each iteration step $n$ so as to minimize the normalized cost functional $\mathcal{F}_{n}\left(\chi_{n}, E_{l, n}\right)$ given by

$$
\mathcal{F}_{n}\left(\chi_{n}, E_{l, n}\right)=W_{\Omega} \sum_{l=1}^{N_{s}}\left\|h_{l, n}^{(1)}\right\|_{\Omega}^{2}+W_{\Gamma} \sum_{l=1}^{N_{s}}\left\|h_{l, n}^{(2)}\right\|_{\Gamma}^{2}
$$

where the normalizing coefficients $W_{\Omega}$ and $W_{\Gamma}$ are defined as:

$$
W_{\Omega}=\frac{1}{\sum_{l=1}^{N_{s}}\left\|E_{l}^{\mathrm{inc}}\right\|_{\Omega}^{2}}, \quad W_{\Gamma}=\frac{1}{\sum_{l=1}^{N_{s}}\left\|f_{l}\right\|_{\Gamma}^{2}} .
$$

Subscripts $\Gamma$ and $\Omega$ are included in the norm $\|\cdot\|$ and later in the inner product $\langle\cdot, \cdot\rangle$ to indicate the domain of integration. The functions $h_{l, n}^{(1)}$ and $h_{l, n}^{(2)}$ are two residual errors. The first one is the residual error with respect to the incident field in the test domain computed from 
the coupling equation. The second residual error is the error on the scattered field computed from the observation equation

$$
\begin{aligned}
& h_{l, n}^{(1)}=E_{l}^{\mathrm{inc}}-E_{l, n}+\mathbf{G}_{\Omega} \chi_{n} E_{l, n}, \\
& h_{l, n}^{(2)}=f_{l}-\mathbf{G}_{\Gamma} \chi_{n} E_{l, n} .
\end{aligned}
$$

The use of a priori information may improve the results of the inversion algorithm. For instance, a binary constraint [16] or positivity constraint $[13,15]$ is used to retrieve the shape of a homogeneous object with known constitutive parameters. In the present work, we incorporate a priori information stating that both real and imaginary parts of the susceptibility $\zeta$ of the target are non-negative $\left(\varepsilon_{r}(\boldsymbol{r})=1+\zeta(\boldsymbol{r})\right)$. Instead of retrieving a complex function $\chi_{n}$, two real auxiliary functions $\xi_{n}$ and $\eta_{n}$ are reconstructed such that

$$
\chi_{n}=1+\xi_{n}^{2}+\mathrm{i} \eta_{n}^{2}-\varepsilon_{2 r} .
$$

The recursive relation with respect to contrast $\chi_{n}$ (equation (7)) becomes

$$
\begin{aligned}
& \xi_{n}=\xi_{n-1}+\beta_{n ; \xi} d_{n ; \xi} \\
& \eta_{n}=\eta_{n-1}+\beta_{n ; \eta} d_{n ; \eta} .
\end{aligned}
$$

Once the updating directions $d_{n ; \xi}, d_{n ; \eta}, v_{n, l}$ and $w_{l, n}$ are found, $\mathcal{F}_{n}$ is a non-linear expression with $2 N_{s}$ complex variables $\left(\alpha_{l, n ; v}, \alpha_{l, n ; w}\right)$ and two real variables $\left(\beta_{n ; \xi}, \beta_{n ; \eta}\right)$. The minimization of $\mathcal{F}_{n}$ is accomplished using the Polak-Ribière conjugate gradient method [17].

\subsection{Search directions}

As the updating directions $d_{n ; \xi}$ and $d_{n ; \eta}$ the authors take the standard Polak-Ribière conjugate gradient directions $[1,15]$. These directions read

$$
\begin{aligned}
& d_{n ; \xi}=g_{n ; \xi}+\gamma_{n ; \xi} d_{n-1 ; \xi}, \quad \text { with } \quad \gamma_{n ; \xi}=\frac{\left\langle g_{n ; \xi}, g_{n ; \xi}-g_{n-1 ; \xi}\right\rangle_{\Omega}}{\left\|g_{n-1 ; \xi}\right\|_{\Omega}^{2}}, \\
& d_{n ; \eta}=g_{n ; \eta}+\gamma_{n ; \eta} d_{n-1 ; \eta}, \quad \text { with } \quad \gamma_{n ; \eta}=\frac{\left\langle g_{n ; \eta}, g_{n ; \eta}-g_{n-1 ; \eta}\right\rangle_{\Omega}}{\left\|g_{n-1 ; \eta}\right\|_{\Omega}^{2}},
\end{aligned}
$$

where $g_{\xi}$ (respectively $\left.g_{\eta}\right)$ is the gradient of the cost functional $\mathcal{F}_{n}\left(\xi, \eta, E_{l}\right)$ with respect to $\xi$ (respectively $\eta$ ), evaluated at the $(n-1)$ th step, assuming that the total field inside the test domain does not change. These gradients are given by

$$
\begin{aligned}
& g_{n ; \xi}=2 \xi_{n-1} \operatorname{Re}\left[W_{\Omega} \sum_{l=1}^{N_{s}} \bar{E}_{l, n-1} \mathbf{G}_{\Omega}^{\dagger} h_{l, n-1}^{(1)}-W_{\Gamma} \sum_{l=1}^{N_{s}} \bar{E}_{l, n-1} \mathbf{G}_{\Gamma}^{\dagger} h_{l, n-1}^{(2)}\right], \\
& g_{n ; \eta}=2 \eta_{n-1} \operatorname{Im}\left[W_{\Omega} \sum_{l=1}^{N_{s}} \bar{E}_{l, n-1} \mathbf{G}_{\Omega}^{\dagger} h_{l, n-1}^{(1)}-W_{\Gamma} \sum_{l=1}^{N_{s}} \bar{E}_{l, n-1} \mathbf{G}_{\Gamma}^{\dagger} h_{l, n-1}^{(2)}\right],
\end{aligned}
$$

where the overbar denotes the complex conjugate, and $\mathbf{G}_{\Omega}^{\dagger}$ and $\mathbf{G}_{\Gamma}^{\dagger}$ are the adjoint operators of $\mathbf{G}_{\Omega}$ and $\mathbf{G}_{\Gamma}$, respectively. 
The first search direction $v_{l, n}$ for the total field inside the test domain is similar to those chosen for the object functions $\xi$ and $\eta$ :

$v_{l, n}=g_{l, n ; E}+\gamma_{l, n ; E_{l}} v_{l, n-1}, \quad$ with $\quad \gamma_{l, n ; E_{l}}=\frac{\left\langle g_{l, n ; E_{l}}, g_{l, n ; E_{l}}-g_{l, n-1 ; E_{l}}\right\rangle_{\Omega}}{\left\|g_{l, n-1 ; E_{l}}\right\|_{\Omega}^{2}}$,

where $g_{l, n ; E_{l}}$ is the gradient of the cost functional $\mathcal{F}_{n}\left(\xi, \eta, E_{l}\right)$ with respect to the field $E_{l}$, evaluated at the $(n-1)$ th step and assuming that $\xi$ and $\eta$ do not change. We obtain

$$
g_{l, n ; E_{l}}=W_{\Omega}\left[\bar{\chi}_{n-1} \mathbf{G}_{\Omega}^{\dagger} h_{l, n-1}^{(1)}-h_{l, n-1}^{(1)}\right]-W_{\Gamma} \bar{\chi}_{n-1} \mathbf{G}_{\Gamma}^{\dagger} h_{l, n-1}^{(2)} .
$$

\subsection{Initial estimates: back-propagation technique}

Given the a priori information stating that the object function is positive, the initial guess $\left(\xi_{0}=0 ; \eta_{0}=0\right)$ must be rejected since the gradients vanish $\left(g_{1 ; \xi}=0 ; g_{1 ; \eta}=0\right)$. This is caused by the local maximum of the cost function introduced by the transformation (13). Thus, we need another initial guess. This can be provided by the back-propagation method $[4,15,16]$.

\subsection{The DORT method}

In the presence of clutter, an efficient way to improve the signal-to-clutter ratio consists in focusing an incident wave onto the scatterer. This can be achieved using the DORT method [3], which provides the set of complex amplitudes needed to synthesize such a wave with the transmitters. In [18], with the same configuration as in the present paper, a comparison between this focusing wave and the field radiated by a single electric dipole has clearly shown an improvement in terms of the signal-to-clutter ratio.

The DORT method is based on the properties of a so-called time-reversal operator. Let $\mathbf{K}$ denote the $N_{s} \times N_{s}$ matrix such that $\mathbf{K}_{i j}$ represents the scattered field recorded by the receiver $j$ when the transmitter $i$ is emitting. Assuming that the embedding medium is lossless, the time reversal experiment is described by the adjoint matrix $\mathbf{K}^{\dagger}$, and the time reversal operator is defined by $\mathbf{L}=\mathbf{K K}^{\dagger}$. The relevant information about the scatterers (number, brightness, localization) is contained in the eigenvalues and eigenvectors of $\mathbf{L}$. One can note that the DORT method requires the same multi-static data as standard inversion techniques.

Expanding the incident and scattered fields as Fourier-Bessel series, one can define the scattering matrix as the linear operator linking the scattering coefficients to the incident ones. It has been shown that the eigenvalues of $\mathbf{L}$ are closely linked to the elements of the scattering matrix $[18,19]$, and that the relationship depends on the geometry of the set-up. When the antennas are located along a line parallel to the $x$ axis, even and odd functions of $x$ form two stable orthogonal subspaces of $\mathbf{L}$. If the angle $\theta$ in the Fourier-Bessel expansion is defined as $\tan \theta=y / x$, writing $\exp (\mathrm{in} \theta)$ as $\cos (n \theta)+\mathrm{i} \sin (n \theta)$ provides a decomposition into even and odd basis functions of the scattered field, and permits one to write $\mathbf{K}$ as the superposition of a symmetric matrix and an antisymmetric matrix. When $\mathbf{L}$ is diagonalized, from low frequency up to the resonance domain, for both TM and TE polarizations, it appears that [18]:

- in each subspace, for each scatterer, one eigenvalue is strongly dominant;

- the even eigenvector attached to the highest eigenvalue can be used to synthesize a wave focusing onto the associated scatterer. 
At low frequencies, in TM polarization, the highest 'even' eigenvalue is larger than the highest 'odd' eigenvalue, since the scatterer behaves almost like a single electric dipole in this case, thus radiating Green's function (even function of $x$ ). In this paper, the DORT method is generally used under such conditions. Therefore, to roughly sum up, the DORT method allows us to extract the dipolar contribution from each scatterer and provides a means of illuminating each one in a selective fashion by backpropagation of this sole contribution.

\subsection{Modification of the inversion algorithm}

Let $E_{l}^{\text {inc;DORT }}$ denote the synthesized incident field focusing onto the scattered number $l=1, \ldots, N$ and by $f_{l}^{\text {DORT }}$ the corresponding scattered field. The scattered field $f_{l}^{\text {DORT }}$ can be derived from the measured data $f_{l}$ through a linear combination of lines of $\mathbf{K}$ in accordance with the components of the associated eigenvector. Our suggestion here is to include, in the iterative scheme, as described in subsection 3.1, $N$ extra incident fields and $N$ extra scattered fields such that the contrast and the total fields in the test domain $\Omega$ are determined iteratively by minimizing a cost functional of the form

$$
\tilde{\mathcal{F}}_{n}\left(\chi_{n}, E_{n}, E_{n}^{\mathrm{DORT}}\right)=\mathcal{F}_{n}\left(\chi_{n}, E_{n}\right)+v^{2} \mathcal{F}_{n}^{\mathrm{DORT}}\left(\chi_{n}, E_{n}^{\mathrm{DORT}}\right) .
$$

The field quantity $E^{\text {DORT }}$ represents the total field in the scattering domain $\Omega$ related to the incident field $E^{\text {inc;DORT }}$. In practice, the positive weight $v^{2}$ is determined, such that $\mathcal{F}$ and $\mathcal{F}^{\text {DORT }}$ are of the same order of magnitude. One way to circumvent the intuitive choice of the weight $v^{2}$ is to introduce the information derived from the DORT method as a multiplicative contribution. In this case, the minimized cost functional is of the form

$$
\tilde{\mathcal{F}}_{n}\left(\chi_{n}, E_{n}, E_{n}^{\mathrm{DORT}}\right)=\mathcal{F}_{n}\left(\chi_{n}, E_{n}\right) \times \mathcal{F}_{n}^{\mathrm{DORT}}\left(\chi_{n}, E_{n}^{\mathrm{DORT}}\right) .
$$

The cost functional $\mathcal{F}_{n}$ is unchanged from the one defined in subsection 3.1 , while the new cost functional $\mathcal{F}^{\text {DORT }}$ reads

$\mathcal{F}_{n}^{\mathrm{DORT}}\left(\chi_{n}, E_{l, n}^{\mathrm{DORT}}\right)=W_{\Omega}^{\mathrm{DORT}} \sum_{l=1}^{N}\left\|h_{l}^{(1 ; \mathrm{DORT})}\right\|_{\Omega}^{2}+W_{\Gamma}^{\mathrm{DORT}} \sum_{l=1}^{N}\left\|h_{l}^{(2 ; \mathrm{DORT})}\right\|_{\Gamma}^{2}$,

where the residual errors $h^{(1 ; \text { DORT })}$ and $h^{(2 ; \text { DORT })}$ are defined similar to $h^{(1)}$ and $h^{(2)}$, respectively.

$$
\begin{aligned}
& h_{l}^{(1 ; \mathrm{DORT})}=E_{l}^{\mathrm{inc} ; \mathrm{DORT}}-E_{l, n}^{\mathrm{DORT}}+\mathbf{G}_{\Omega} \chi_{n} E_{l, n}^{\mathrm{DORT}}, \\
& h_{l}^{(2 ; \mathrm{DORT})}=f_{l}^{\mathrm{DORT}}-\mathbf{G}_{\Gamma} \chi_{n} E_{l, n}^{\mathrm{DORT}} .
\end{aligned}
$$

The normalizing coefficients $W_{\Omega}^{\text {DORT }}$ and $W_{\Gamma}^{\text {DORT }}$ are as follows:

$$
W_{\Omega}^{\mathrm{DORT}}=\frac{1}{\sum_{l=1}^{N}\left\|E_{l}^{\mathrm{inc} ; \mathrm{DORT}}\right\|_{\Omega}^{2}}, \quad W_{\Gamma}^{\mathrm{DORT}}=\frac{1}{\sum_{l=1}^{N}\left\|f_{l}^{\mathrm{DORT}}\right\|_{\Gamma}^{2}} .
$$

The updating directions are taken to be of the same type as the ones described previously, in which the gradients involved now pertain to the total cost functional $\tilde{\mathcal{F}}$ instead of $\mathcal{F}$. Derivation of these gradients is straightforward in both additive and multiplicative schemes and therefore the expressions of these gradients are not written herein. Note that the cost function $\tilde{\mathcal{F}}_{n}$ that is minimized at each iteration step is now a polynomial of $2\left(N_{s}+N\right)$ complex variables and still two real variables $\left(\beta_{n ; \xi}, \beta_{\eta ; \eta}\right)$. 


\section{Numerical experiments}

In this section, we discuss reconstruction results using synthetic data. These data have been generated using a forward solver based on the conjugate-gradient method with the aid of fast Fourier transforms in two different configurations. The first one involves a dielectric object is buried in a homogeneous half-space. The second one corresponds to the same object but buried in a cluttered environment. The unknown object is a homogeneous dielectric circular cylinder of relative permittivity $\varepsilon_{r}=7$ and radius $a=0.1 \mathrm{~m}$ buried in a medium of relative permittivity $\varepsilon_{2 r}=4$. The upper medium is of permittivity $\varepsilon_{1}=\varepsilon_{0}$. The centre of the cylinder is at $0.75 \mathrm{~m}$ depth and shifted from the centre of the segment of line $\Gamma$ by a distance of $0.1 \mathrm{~m}$. Along the line $\Gamma, N_{s}=N_{r}=31$ antennas are regularly distributed. Each antenna plays the role of emitter and receiver. The length of the measurement line $\Gamma$ is $6 \mathrm{~m}$ and three operating frequencies are considered: $f=100,200$ and $300 \mathrm{MHz}$. The cluttered data were obtained with the cylinder embedded in a large $(3 \times 3) \mathrm{m}^{2}$ box, discretized into $128 \times 128$ cells, where the permittivity in cells situated outside the cylinder randomly fluctuates, with uniform distribution, between $(1-b) \varepsilon_{2}$ and $(1+b) \varepsilon_{2}$, with $b=20 \%$ being the amplitude of the fluctuations. Clutter is quantified via a single number, err, defined as

$$
e r r=\sqrt{\frac{\sum_{l=1}^{L}\left\|f_{l}^{s}-f_{l}\right\|_{\Gamma}^{2}}{\sum_{l=1}^{L}\left\|f_{l}\right\|_{\Gamma}^{2}}},
$$

where $f^{s}$ and $f$ denote the scattered field with and without clutter, respectively. For the reported results, the value of err is 33, 49 and $129 \%$ at $f=100,200$ and $300 \mathrm{MHz}$, respectively.

All reported reconstruction results correspond to the 32nd iteration with the test domain $\Omega$ of size $(1 \times 1) \mathrm{m}^{2}$ discretized into $31 \times 31$ cells, which leads to a different discretization than the one used to generate the data. Note that the size of $\Omega$ is nine times smaller than the box used to generate the cluttered data. We did not observe any change by continuing the iterative process further. In all examples, only the real part of the complex permittivity is displayed. In fact, the reconstructed conductivity distribution was, in almost all cases, null and therefore results of the reconstruction of the conductivity are not given. We did not use the a priori information that the target under test is dielectric, although using this information may have slightly improved the results of the reconstruction but would not have changed our conclusions.

\subsection{Results at fixed frequency}

In order to provide an efficient strategy for the inversion, we herein examine the ideal case of noiseless data. Figure 2 presents the reconstruction results at fixed frequency without taking information derived from DORT into account. In these results, the initial guess was deduced from the back-propagation procedure. Note the improvement in the quality of the reconstruction when switching the operating frequency from $f=100 \mathrm{MHz}$ to $200 \mathrm{MHz}$ but deterioration of the resolution at $f=300 \mathrm{MHz}$. This shows the sensitivity of the choice of the initial estimate. The dynamic range as well as the resolution aspects of iterative inverse scattering schemes were analysed in [20], leading to the idea of the multiple-frequency approach, or the frequency-hopping approach [21].

\subsection{Reconstruction using the multiple-frequency approach}

From what precedes, we conclude that reconstructing at fixed frequencies starting from a back-propagation initial is not an efficient strategy. We examine now the multiple-frequency 


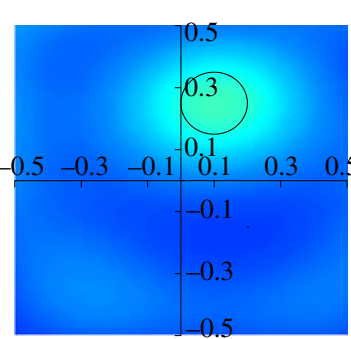

(a)

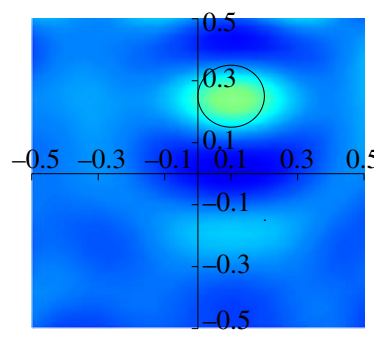

(d)

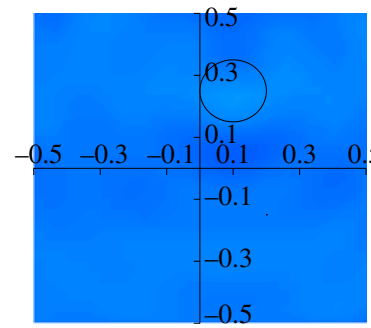

(g)

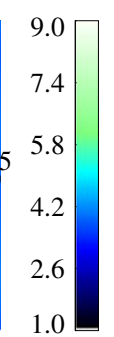

1.0

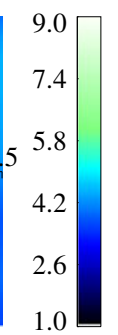

1.0

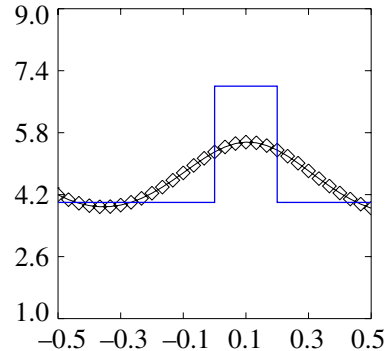

(b)

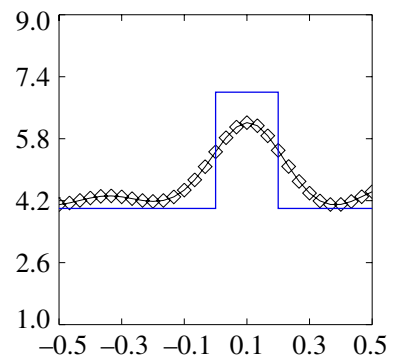

(e)
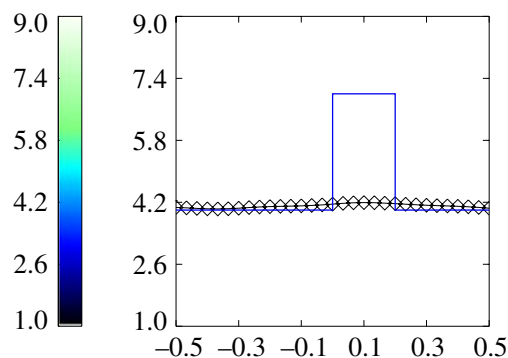

(h)

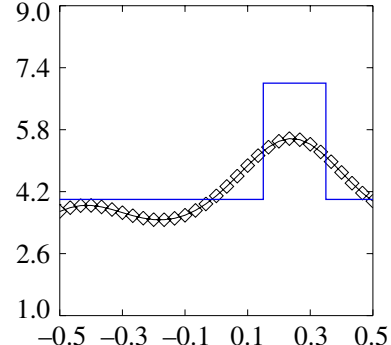

(c)

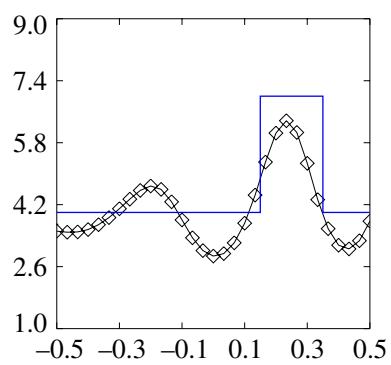

(f)

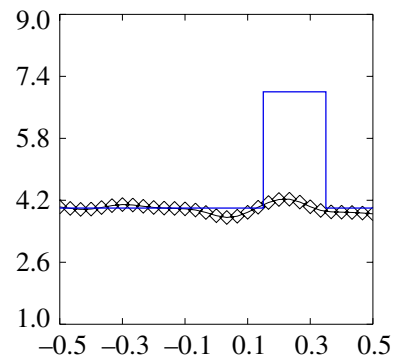

(i)

Figure 2. Reconstructed permittivity distribution from noiseless data at fixed frequencies: $f=$ $100 \mathrm{MHz}$ (a), $200 \mathrm{MHz}$ (d) and $300 \mathrm{MHz}$ (g). The circles plotted in (a), (d) and (g) represent the boundary of the actual cylinder. Comparison between the reconstructed ( $\square$ ) and actual profiles (-) along vertical and horizontal lines crossing the centre of the cylinder are plotted in the middle and right columns, respectively.

approach, i.e., using as the initial guess the final result obtained at the immediately lower frequency. Figure 3 shows the results of the reconstruction using this approach and exhibits significant improvement, as compared to figure 2.

\subsection{Reconstruction of a target buried in a highly cluttered environment}

Consider now the case of the cylinder with clutter. Figures 4(a) and (b) present results of reconstruction of the cylinder when the clutter is present, to be compared to the results from noiseless data in figures 4(c) and (d), all being obtained using the frequency-hopping approach. From these results, we conclude that the strategy based on the multiple-frequency approach is not sufficiently efficient to reconstruct the target buried in a highly cluttered environment. Other ways must be explored. 


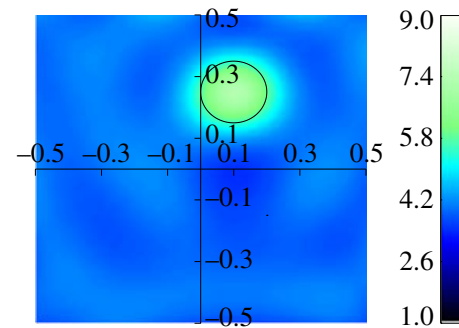

(a)

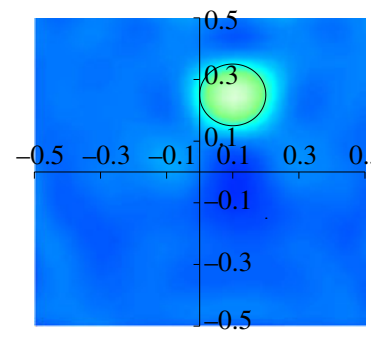

(d)

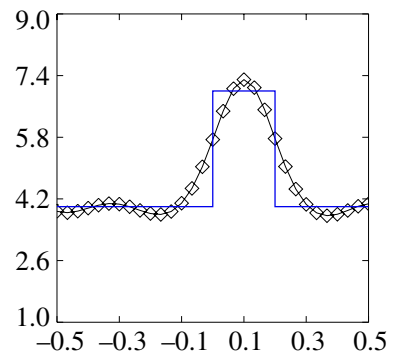

(b)
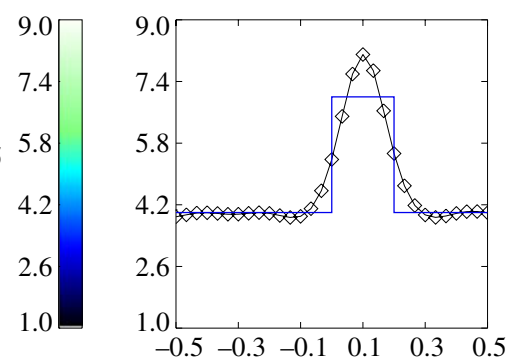

(e)

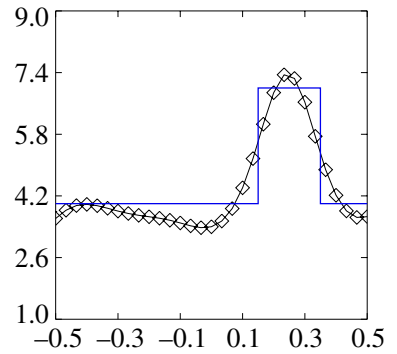

(c)

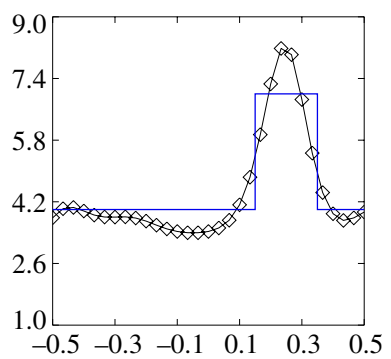

(f)

Figure 3. Reconstructed permittivity distributions from noiseless data using the multiple frequency approach (frequency hopping approach). (a) Result at $f=200 \mathrm{MHz}$ with the final result at $f=100 \mathrm{MHz}$ as initial guess; (d) result at $f=300 \mathrm{MHz}$ with (a) as initial estimate. Comparison between the reconstructed profile $(\square)$ and the actual one ( - ) along vertical and horizontal lines crossing the centre of the target are plotted in the middle and right columns, respectively.

\subsection{Use of the DORT method in the inverse algorithm}

To enhance the target contribution, one might use the focusing incident field derived from the DORT method, as described in subsection 3.5. Figure 5 shows a map of the field of the focusing wave, derived from the eigenvector associated with the highest eigenvalue, which concentrates energy around $x=0.1$ and permits us to define the area of interest for inversion. Figure 6 presents results of the reconstruction using the information derived from DORT with the frequency-hopping approach. We report both additive, with weight parameter $v^{2}=6$, and multiplicative ways of taking into account the synthesized fields plotted in figure 5 . Comparison with results obtained without the DORT method (figures 4(a) and (b)) shows some improvement, especially at $f=300 \mathrm{MHz}$ when clutter generates err as high as $129 \%$. However, some oscillations remain and prevent the target from being extracted from the clutter. In our opinion, such oscillations are linked to the stratified geometry, which, combined with the aspect-limited configuration, provides many solutions to the inverse problem. This is discussed in the next subsection.

\subsection{Non-uniqueness of the solution}

Let us consider an incident beam, described as a superposition of plane waves impinging from the upper medium onto the interface, with $a(\alpha)$ denoting the complex amplitude of the plane wave with horizontal wavenumber $\alpha$ at $y=0^{+}$. If $t_{d}(\alpha)$ is the complex transmission coefficient when this plane wave is propagating downwards ( $t_{u}$ for upgoing waves), the field transmitted 


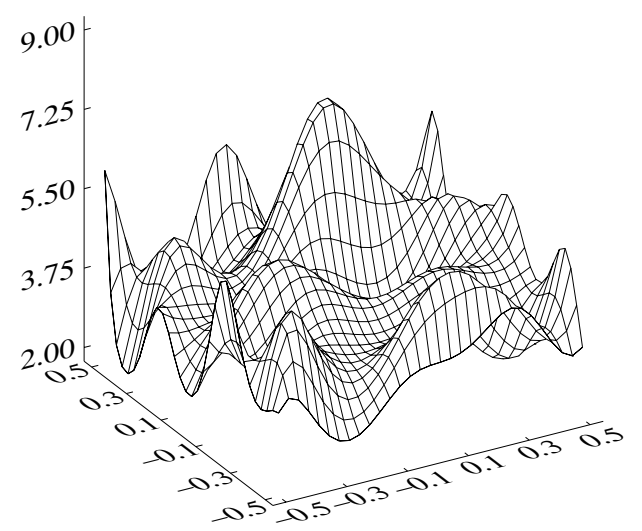

(a)

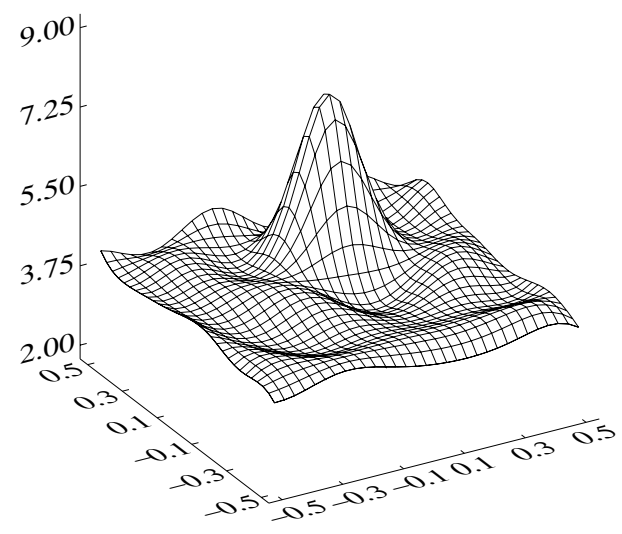

(c)

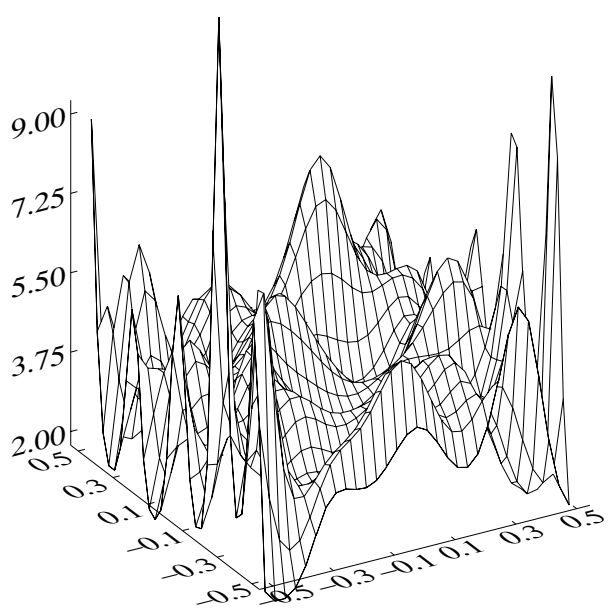

(b)

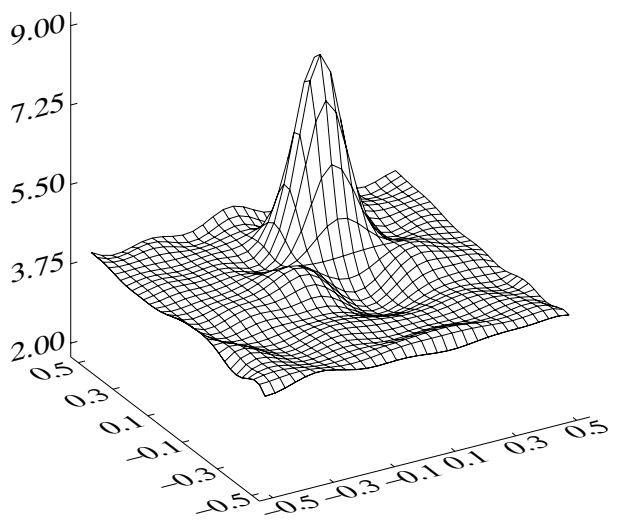

(d)

Figure 4. Reconstructed permittivity using the multiple frequency approach with ((a), (b)) and without ((c), (d)) clutter. Results at $f=200 \mathrm{MHz}$ with final reconstruction obtained at $f=$ $100 \mathrm{MHz}$ as initial guess plotted in (a) and (c). Results at $f=300 \mathrm{MHz}$ with (a) and (c) as initial estimates are plotted in (b) and (d), respectively.

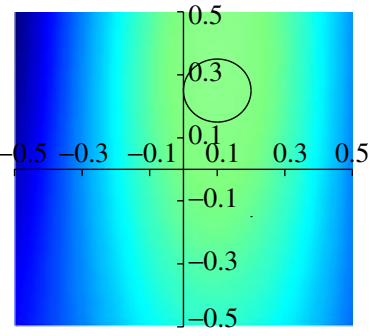

(a)

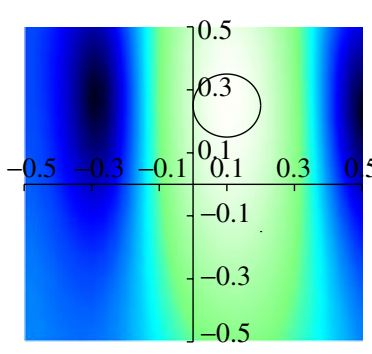

(b)

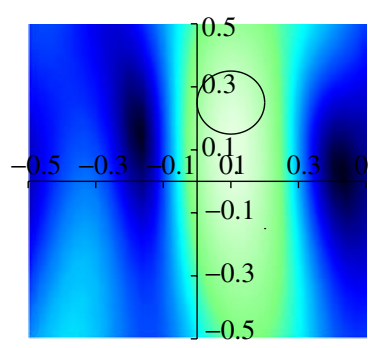

(c)

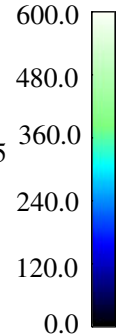

0.0

Figure 5. Map of the modulus of the synthetized field focusing onto the target deduced from the DORT method and utilized as extra incident field in the inversion algorithm. (a) $f=100 \mathrm{MHz}$; (b) $f=200 \mathrm{MHz}$ and (c) $f=300 \mathrm{MHz}$. 


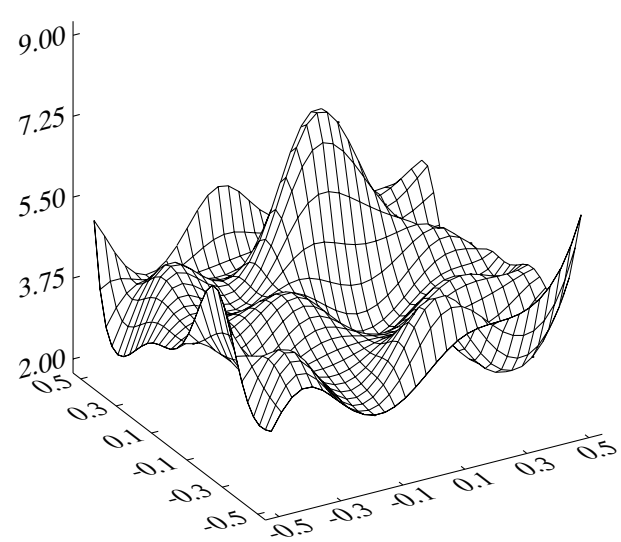

(a)

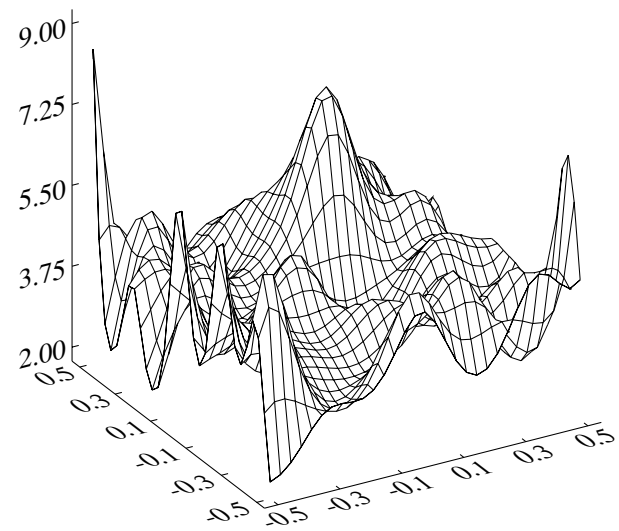

(c)

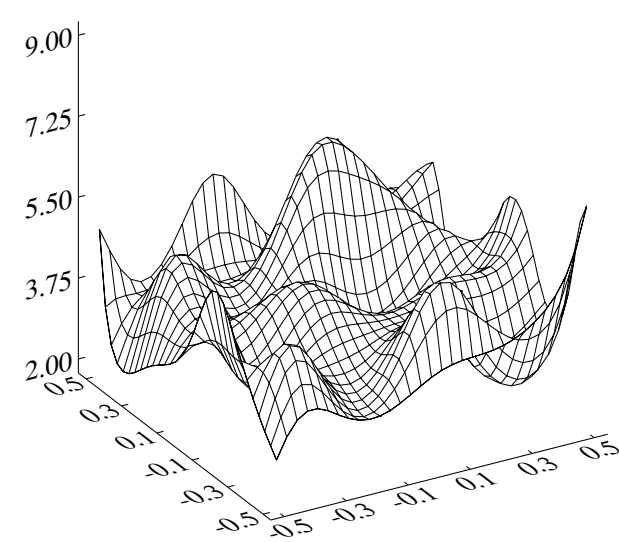

(b)

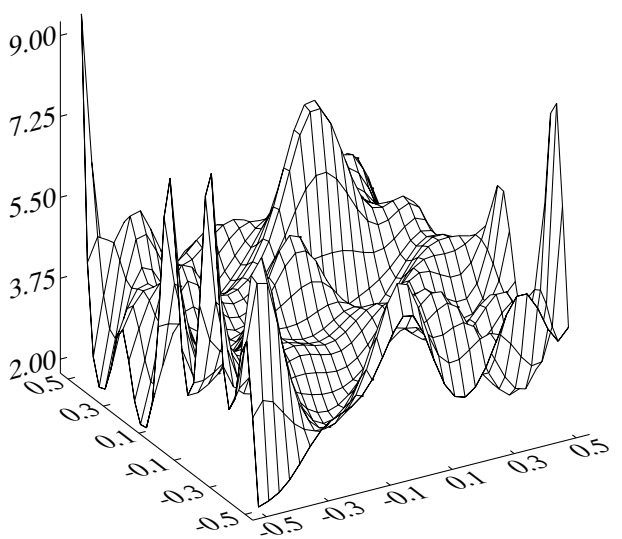

(d)

Figure 6. Reconstructed permittivity distributions, using the multiple frequency approach, from cluttered data. Inversion is carried out by including information derived from the DORT method in the iterative process. ((a), (c)) Results at $f=200$ and $300 \mathrm{MHz}$, respectively, using the multiplicative framework to incorporate the information derived from the DORT method. ((b), (d)) Results at $f=200$ and $300 \mathrm{MHz}$, respectively, using the additive framework to incorporate the information derived from the DORT method.

in the lower medium is given by

$$
E^{t}(x, y)=\int \mathrm{d} \alpha a(\alpha) t_{d}(\alpha) \exp \left(\mathrm{i} \alpha x-\mathrm{i} \beta_{2} y\right)
$$

$\beta_{j}=\left(k_{j}^{2}-\alpha^{2}\right)^{1 / 2}, \operatorname{Re}\left(\beta_{j}\right)+\operatorname{Im}\left(\beta_{j}\right) \geqslant 0, j=1,2$. In the frame of the Born approximation, the field scattered in the upper medium by a buried object whose support is included in domain $\Omega$ becomes

$E^{s}(x, y) \simeq \mathrm{i} \pi k_{0}^{2} \iint \mathrm{d} \alpha \mathrm{d} \alpha^{\prime} \frac{a(\alpha)}{\beta_{2}^{\prime}} t_{d}(\alpha) t_{u}\left(\alpha^{\prime}\right) \widehat{\chi}\left(\alpha^{\prime}-\alpha, \beta_{2}+\beta_{2}^{\prime}\right) \exp \left(\mathrm{i} \alpha^{\prime} x+\mathrm{i} \beta_{1}^{\prime} y\right)$, 
where the Fourier transform of $f(x, y)$ is defined as

$$
\widehat{f}(\alpha, \beta)=\iint \frac{\mathrm{d} x}{2 \pi} \frac{\mathrm{d} y}{2 \pi} f(x, y) \exp (-\mathrm{i} \alpha x-\mathrm{i} \beta y) .
$$

When both transmitter and receiver are not located very close to the interface, evanescent waves are not involved in the scattering process, thus $a(\alpha)$ and $\exp \left(\mathrm{i} \beta_{1}^{\prime} y\right)$ rapidly tend to zero when $|\alpha|$ and $\left|\alpha^{\prime}\right|$ become greater than $k_{1}$, respectively. In addition, if $k_{2} \gg k_{1}, \beta_{2}^{(\prime)}$ remains close to $k_{2}$ for $\left|\alpha^{(\prime)}\right|<k_{1}$, and the scattered field can be approximated by

$$
E^{s}(x, y) \simeq \mathrm{i} \pi k_{0}^{2} \iint \mathrm{d} \alpha \mathrm{d} \alpha^{\prime} \frac{a(\alpha)}{\beta_{2}^{\prime}} t_{d}(\alpha) t_{u}\left(\alpha^{\prime}\right) \widehat{\chi}\left(\alpha^{\prime}-\alpha, 2 k_{2}\right) \exp \left(\mathrm{i} \alpha^{\prime} x+\mathrm{i} \beta_{1}^{\prime} y\right) .
$$

In the present paper, $k_{2}=2 k_{1}$ and $\beta_{2}$ decreases from $k_{2}$ to $(\sqrt{3} / 2) k_{2}$ when $\alpha$ increases from 0 to $k_{1}$. Therefore, two contrast functions $\chi$ with the same $y$-Fourier component $\widehat{\chi}\left(., 2 k_{2}\right)$ provide almost the same scattered field, leading to the same kind of ambiguity as in 1D problems. This is obvious in the simple case of a point-like scatterer at depth $d$, described by $\chi(x, y)=\sigma \chi \delta(x, y+d)$, where $\sigma$ is the actual area of the cross-section of the scatterer; equation (30) becomes

$$
E^{s}(x, y) \simeq k_{0}^{2} \sigma \chi \exp \left(2 \mathrm{i} k_{2} d\right) \iint \mathrm{d} \alpha \mathrm{d} \alpha^{\prime} \frac{a(\alpha)}{4 \mathrm{i} \pi \beta_{2}^{\prime}} t_{d}(\alpha) t_{u}\left(\alpha^{\prime}\right) \exp \left(\mathrm{i} \alpha^{\prime} x+\mathrm{i} \beta_{1}^{\prime} y\right)
$$

and clearly shows that changing $\chi$ into $-\chi$ and $d$ into $d \pm \lambda_{2} / 2$ does not modify $E^{s}$. It is easily proven that the same transformation as applied to a homogeneous layer, illuminated under normal incidence, also leaves the reflected field invariant. Indeed, in this case, $\chi(x, y)=\chi \Pi(y+d / h)$, where $\Pi$ is the window function, $a(\alpha)=\delta(\alpha)$ and the scattered field does not depend on $x$, as a result of invariance of the scattering problem with respect to translations along the $x$ direction. Consequently, $E^{s}$ in equation (30) reduces to a reflected plane wave propagating along the vertical axis

$$
\begin{aligned}
E^{s}(y) & =\mathrm{i} \pi \frac{k_{0}^{2}}{k_{2}} t_{d} t_{u} \widehat{\chi}\left(2 k_{2}\right) \exp \left(\mathrm{i} k_{1} y\right) \\
& =\frac{\mathrm{i}}{2} \frac{k_{0}^{2}}{k_{2}^{2}} t_{d} t_{u} \chi \exp \left(2 \mathrm{i} k_{2} d\right) \sin \left(k_{2} h\right) \exp \left(\mathrm{i} k_{1} y\right) . \quad \text { QED }
\end{aligned}
$$

In figure 2 , the shape of the vertical fluctuations of the reconstructed permittivity is well approximated by the Bessel function $J_{0}\left(2 k_{2}\left(y^{\prime}+d\right)\right.$ ), which provides almost the same scattered field as the localized homogeneous object. Analytical calculations in the 1D case, which are much easier to perform, show that a modulated medium with contrast $\chi^{\prime} \Pi\left(\left(y^{\prime}+d\right) / H\right) \cos \left(2 k_{2}\left(y^{\prime}+d\right)\right), k_{2} H \gg 1$, may have the same echo as the previous single homogeneous layer. Indeed, equation (31) becomes

$$
E^{s}(x, y) \simeq \frac{\mathrm{i}}{2} \frac{k_{0}^{2}}{k_{2}^{2}} t_{d} t_{u} \frac{k_{2} H}{2} \chi^{\prime} \exp \left(2 \mathrm{i} k_{2} d\right) \exp \left(\mathrm{i} k_{1} y\right)
$$

and coincides with equation (32) if $\chi^{\prime} k_{2} H=2 \chi \sin \left(k_{2} h\right)$. This is quite similar to the $2 \mathrm{D}$ problem under study. Surprisingly, the use of multi-static data in such 'aspect-limited' configurations does not drastically reduce the number of solutions, even though the line of measurement $\Gamma$ is several wavelengths long.

It should also be emphasized that prior knowledge of the sign of $\chi$ can do away with these oscillating solutions. An example is given in the paper by Lambert et al [22], where 


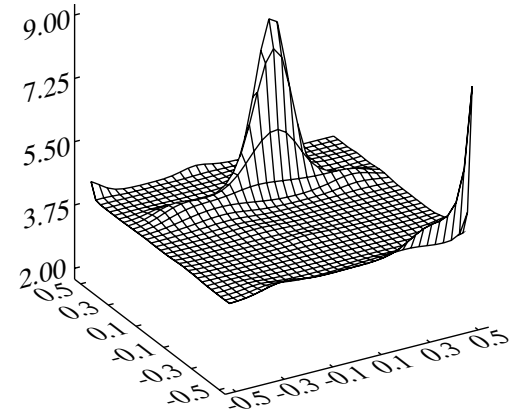

(a)

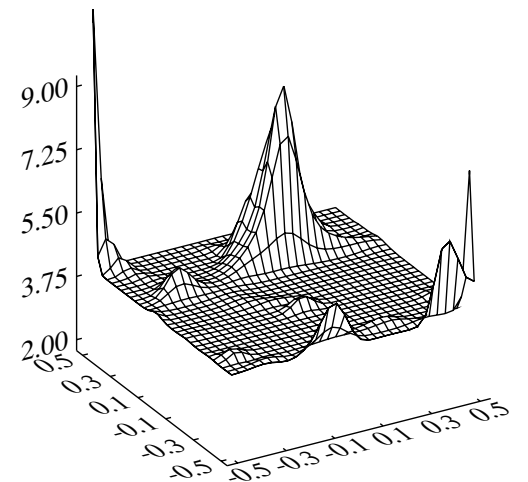

(c)

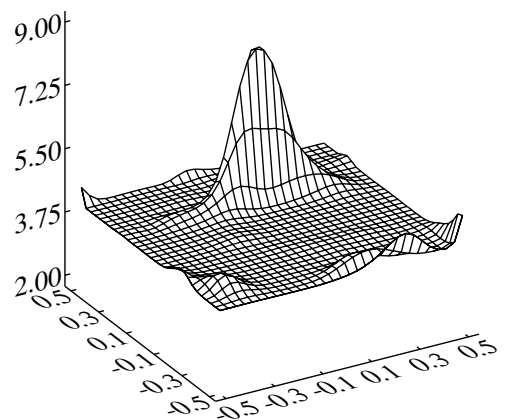

(b)

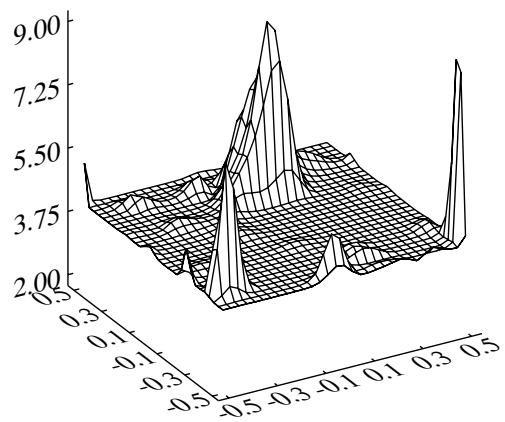

(d)

Figure 7. Same as in figure 6, but with the use of the a priori information that the reconstructed contrast is positive.

the reconstruction of the conductivity of a scatterer buried in a lossless background is very accurate, in contrast to the real part of the permittivity, because positivity is reinforced in the former case, not in the latter. In order to do away with the ambiguity mentioned above, we have included the a priori information stating that the contrast is positive in the inversion scheme. This is done by retrieving the functions $\xi$ and $\eta$ such that (13) is changed to

$$
\chi_{n}=\xi_{n}^{2}+\mathrm{i} \eta_{n}^{2}
$$

which leads to a reconstructed relative permittivity profile of the form $\varepsilon_{r}(\boldsymbol{r})=\varepsilon_{2 r}+\xi(\boldsymbol{r})^{2}+$ i $\eta(\boldsymbol{r})^{2}$. Figure 7 shows the reconstruction results from cluttered data using the DORT method and the frequency hopping approach, to be compared to the ideal case of noiseless data (figures 4(c) and (d)). Note that satisfactory reconstructions are obtained with both additive and multiplicative approaches.

\subsection{Multiple scatterers}

Prior knowledge of the number of disconnected scatterers is not necessary, since a domain formalism is used for inversion. It may happen that the DORT method provides ambiguous information, such that the number of scatterers is underestimated, either because they are too close to each other to be separated at the operating frequency or because their signature is 


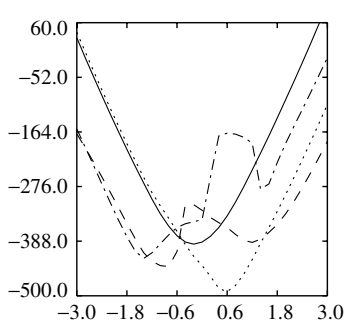

(a)

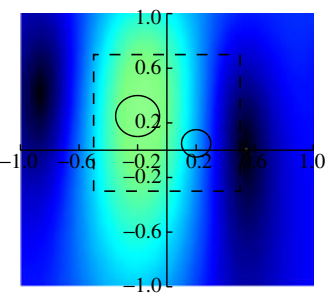

(d)

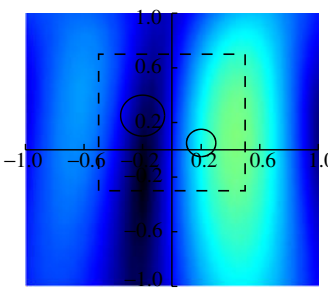

(g)

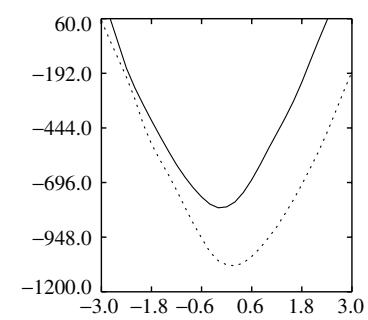

(b)
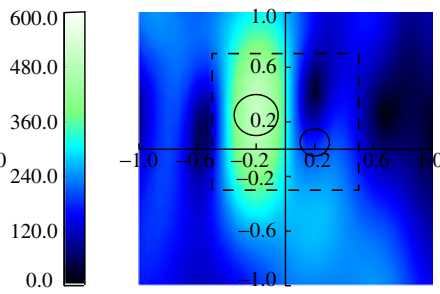

(e)

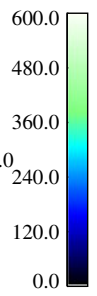

0.0

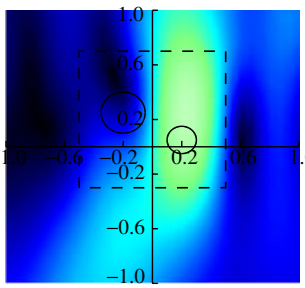

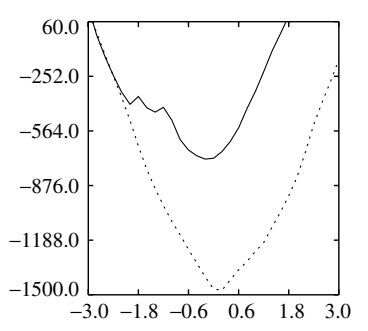

(c)

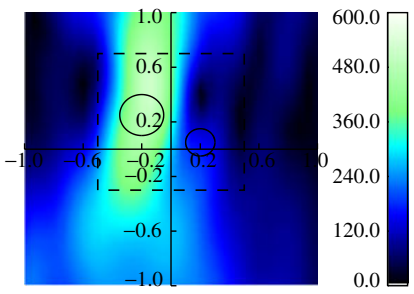

(f)

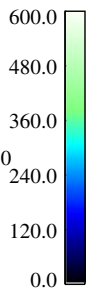

(h)

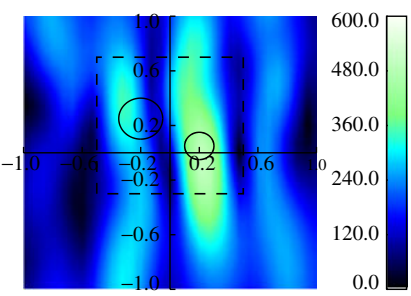

(i)

Figure 8. Frequency increases from left to right: 100, 200 and $300 \mathrm{MHz}$, respectively. Top: phase of the components of the eigenvectors associated with the highest eigenvalues (four at $100 \mathrm{MHz}$, then two). Middle: map of the modulus of the field radiated by a set of fictitious transmitters fed by the components of the eigenvector associated with the highest eigenvalue. Bottom: same as above for the second highest eigenvalue. The circles represent the boundaries of targets under test while the dashed box represents the investigating domain $\Omega$ that is used later in the inversion.

'buried' in clutter. The procedure remains the same however, even if the number of additional incident fields is smaller than the number of targets.

To illustrate the potential of the method, let us consider two scatterers with circular crosssections $\left(r_{1}=0.15 \mathrm{~m}\right.$ and $\left.r_{2}=0.1 \mathrm{~m}\right)$, at depth 0.75 and $0.95 \mathrm{~m}$, shifted from the centre of the line of measurements $\Gamma$ by a distance of -0.2 and $0.2 \mathrm{~m}$, with relative permittivities $\varepsilon_{r}=7$ and 6 , respectively. The amplitude of the fluctuations of the permittivity of the lower medium is $b=0.15$, leading in this case to $e r r=12,28$ and $69 \%$ at $f=100,200$ and $300 \mathrm{MHz}$, respectively.

The phase of the components of the eigenvectors associated with the four highest eigenvalues at $100 \mathrm{MHz}$ is plotted in figure 8(a). Two of them have an axis of symmetry and the other two present a jump, as a signature of anti-symmetric behaviour. Therefore one can conclude that they are associated with two scatterers located below the central part of $\Gamma$. The fifth eigenvalue is 3000 and 200 times smaller than the first and second ones, respectively, and the corresponding eigenvector does not behave as expected (neither even nor odd). Moreover, the behaviour of the two 'highest' eigenvectors remains stable at higher frequencies, with 


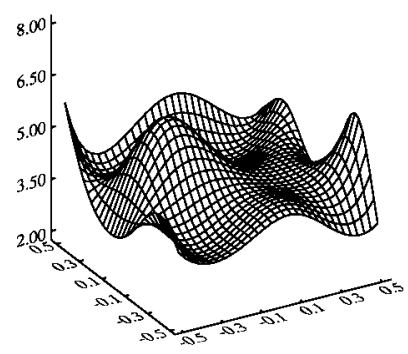

(a)

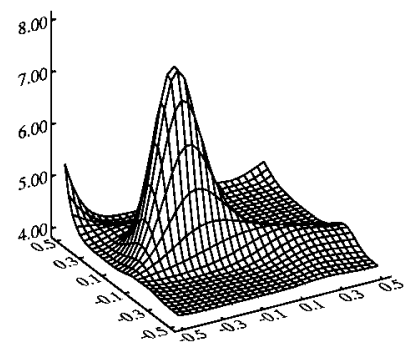

(d)

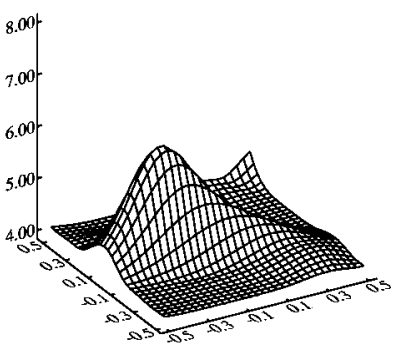

(g)

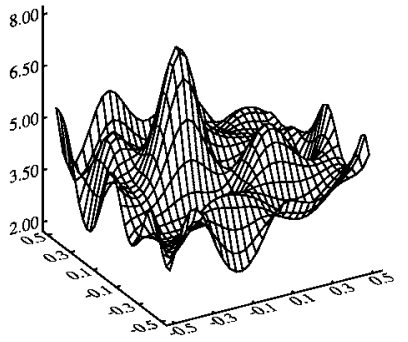

(b)

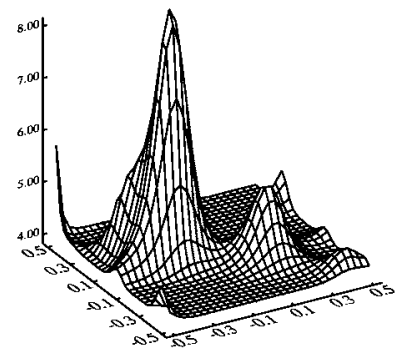

(e)

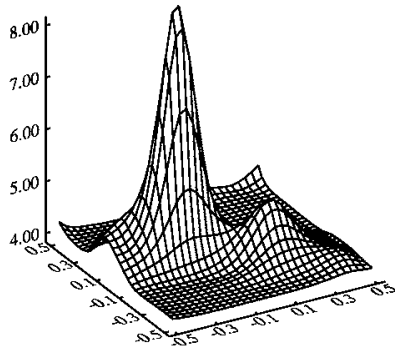

(h)

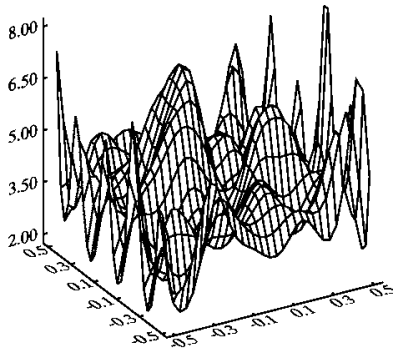

(c)

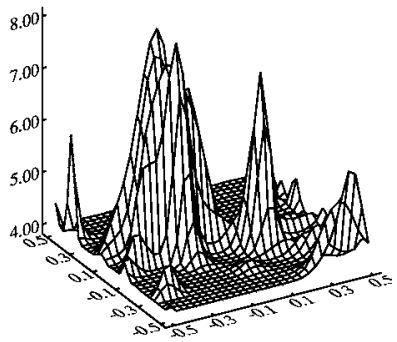

(f)

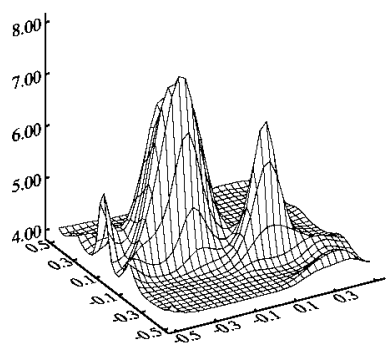

(i)

Figure 9. Reconstructed permittivity distribution using the frequency-hopping approach. Frequency increases from left to right: 100, 200 and $300 \mathrm{MHz}$, respectively. Top: reconstruction without using DORT, nor positivity of the contrast. Middle: reconstruction using both DORT, through an additional term $\left(v^{2}=2.5\right)$ of the cost function, and positivity. Bottom: same as above using DORT through weighting factors in the cost function.

backpropagating waves focusing onto the same areas (figure 8), even though err reaches values as high as $69 \%$ at $300 \mathrm{MHz}$. Two additional incident fields are thus considered in this case, and the reconstructed area is restricted to the 'bright' zones of the focusing fields, namely $[-0.5 \mathrm{~m}, 0.5 \mathrm{~m}] \times[-0.3 \mathrm{~m}, 0.7 \mathrm{~m}]$. In this case, the best reconstruction results are obtained in the multiplicative approach case, as shown in figure 9.

\section{Conclusion}

We have investigated the reconstruction of targets buried in a highly cluttered environment. The inversion was achieved iteratively, using information derived from DORT in order to improve the signal-to-clutter ratio. However, to circumvent ambiguities on the reconstructed profile, inherent to the stratified configuration, a priori information is necessary. When the reconstructed contrast was assumed to be positive, the results were satisfactory. In particular, 
the numerical examples clearly show that clutter rejection resulting from this inversion procedure provides more accurate estimates of both shape and contrast, since, for a given configuration, higher frequencies can be reached. This study also suggests introducing less restrictive a priori information than that used in the paper. This may be achieved by using regularization procedures, for instance.

\section{References}

[1] Belkebir K and Tijhuis A G 2001 Modified $^{2}$ gradient method and modified Born method for solving a twodimensional inverse scattering problem Inverse Problems 17 1671-88

[2] Lesselier D and Duchêne B 1991 Buried, 2-D penetrable objects illuminated by line sources: FFT-based iterative computations of the anomalous field Progr. Electromagn. Res. 5 351-89

[3] Prada C, Manneville S, Spoliansky D and Fink M 1996 Decomposition of the time reversal operator: detection and selective focusing on two scatterers J. Acoust. Soc. Am. 9 2067-76

[4] Belkebir K, Bonnard S, Pezin F, Sabouroux P and Saillard M 2000 Validation of 2D inverse scattering algorithms from multi-frequency experimental data J. Electromagn. Waves Appl. 14 1637-67

[5] Saillard M, Vincent $P$ and Micolau G 2000 Reconstruction of buried objects surrounded by small inhomogeneities Inverse Problems 16 1195-208

[6] Roger A 1981 Newton-Kantorovitch algorithm applied to an electromagnetic inverse problem IEEE Trans. Antennas Propag. 29 232-8

[7] Joachimowicz N, Pichot C and Hugonin J-P 1991 Inverse scattering: an iterative numerical method for electromagnetic imaging IEEE Trans. Antennas Propag. 39 1742-53

[8] Chew W C and Wang Y M 1990 Reconstruction of two-dimensional permittivity distribution using distorted Born iterative method IEEE Trans. Med. Imaging 9 218-25

[9] Tijhuis A G 1989 Born-type reconstruction of material parameters of an inhomogeneous lossy dielectric slab from reflected-field data Wave Motion 11 151-73

[10] Remis R F and van den Berg P M 2000 On the equivalence of the Newton-Kantorovich and distorted Born methods Inverse Problems 16 L1-L4

[11] Kleinman R E and van den Berg P M 1992 A modified gradient method for two-dimensional problems in tomography J. Comput. Appl. Math. 42 17-35

[12] van den Berg P M and Kleinman R E 1997 A contrast source inversion method Inverse Problems 13 1607-20

[13] Belkebir K, Kleinman R E and Pichot C 1997 Microwave imaging-location and shape reconstruction from multifrequency scattering data IEEE Trans. Microwave Theory Tech. 45 469-76

[14] van den Berg P M and Kleinman R E 1995 A total variation enhanced modified gradient algorithm for profile reconstruction Inverse Problems 11 L5-10

[15] Kleinman R E and van den Berg P M 1994 Two-dimensional location and shape reconstruction Radio Sci. 29 $1157-69$

[16] Souriau L, Duchêne B, Lesselier D and Kleinman R E 1996 Modified gradient approach to inverse scattering for binary objects in stratified media Inverse Problems 12 463-81

[17] Press W H, Flannery B P, Teukolski S A and Vetterling W T 1986 Numerical Recipes. The Art of Scientific Computing (Cambridge: Cambridge University Press)

[18] Micolau G and Saillard M 2003 DORT method as applied to electromagnetic sensing of buried objects Radio Sci. 381038

[19] Tortel H, Micolau G and Saillard M 1999 Decomposition of the time reversal operator for electromagnetic scattering J. Electromagn. Waves Appl. 13 687-719

[20] Tijhuis A G, Belkebir K, Litman A C S and de Hon B P 2001 Theoretical and computational aspects of 2-D inverse profiling IEEE Trans. Geoscience Remote Sensing 39 1316-30

[21] Chew W C and Lin J H 1995 A frequency-hopping approach for microwave imaging of large inhomogeneous bodies IEEE Microwave Guided Wave Lett. 5 439-41

[22] Lambert M, Lesselier D and Kooij B J 1998 The retrieval of a buried cylindrical obstacle by a constrained modified gradient method in the H-polarization case and for Maxwellian materials Inverse Problems 14 1265-83 